\title{
TITLE:
}

\section{Adsorbed states and scanning tunneling microscopy induced migration of acetylene on $\mathrm{Cu}(110)$}

$\operatorname{AUTHOR}(S):$

Kumagai, T; Hatta, S; Okuyama, H; Aruga, T

\section{CITATION:}

Kumagai, T ...[et al]. Adsorbed states and scanning tunneling microscopy induced migration of acetylene on Cu(110). JOURNAL OF CHEMICAL PHYSICS 2007, 126(23): 234708.

ISSUE DATE:

2007-06-21

URL:

http://hdl.handle.net/2433/50589

\section{RIGHT:}

Copyright 2007 American Institute of Physics. This article may be downloaded for personal use only. Any other use requires prior permission of the author and the American Institute of Physics. 


\title{
Adsorbed states and scanning tunneling microscopy induced migration of acetylene on $\mathrm{Cu}(110)$
}

\author{
T. Kumagai, S. Hatta, H. Okuyama, ${ }^{\text {a) }}$ and T. Aruga \\ Department of Chemistry, Graduate School of Science, Kyoto University, Kyoto 606-8502, Japan
}

(Received 26 February 2007; accepted 19 April 2007; published online 20 June 2007)

\begin{abstract}
The authors have studied adsorption of acetylene on $\mathrm{Cu}(110)$ by means of low-temperature scanning tunneling microscopy. Adsorbed molecules preferentially aggregate at $40 \mathrm{~K}$ to yield dimer, trimer, and larger islands on the surface. Isolated species (monomer) adsorbs on the fourfold hollow site with $\sim s p^{3}$ rehybridization as characterized by inelastic electron tunneling spectroscopy. Tunneling electron induces an acetylene molecule to migrate along the trough of $\mathrm{Cu}(110)$. The migration proceeds in two steps: the molecule first hops to the adjacent long-bridge site and then to the next fourfold site. The voltage and current dependencies of the hopping probability show that the migration is induced by inelastic electron tunneling that causes vibrational excitation of mainly $\mathrm{C}-\mathrm{H}$ stretch mode. (C) 2007 American Institute of Physics. [DOI: 10.1063/1.2741512]
\end{abstract}

\section{INTRODUCTION}

Scanning tunneling microscopy (STM) has been used not only to image but also to manipulate and characterize individual molecules on surfaces. ${ }^{1}$ The capability of studying a single molecule provides local information such as environmental effect that cannot be obtained from macroscopic measurements. It was first demonstrated that STM can conduct vibrational spectroscopy at single-molecule level by detecting $\mathrm{C}-\mathrm{H}$ stretch vibration of acetylene on $\mathrm{Cu}(100){ }^{2}$ Single-molecule motions such as rotation ${ }^{3}$ and translation ${ }^{4}$ were induced by vibrational excitation with STM.

Adsorption of acetylene on metal surfaces has been studied as a prototypical system of catalytic reaction. It was reported that acetylene trimerization reaction proceeds with almost $100 \%$ efficiency on $\mathrm{Cu}(110)$ under ultrahigh-vacuum condition. ${ }^{5,6}$ The reaction was observed even at low coverage, suggesting island formation of acetylene on $\mathrm{Cu}(110){ }^{6}$ The adsorption site, orientation, and rehybridization were studied by high-resolution electron energy loss spectroscopy ${ }^{5,7}$ and by $\mathrm{x}$-ray absorption/emission spectroscopy with the aid of theoretical calculations. ${ }^{8}$ The vibrational spectroscopy suggested a distorted configuration with $\sim s p^{3}$ rehybridization. $^{7}$ The recent electronic study revealed two molecular states: ${ }^{8}$ one adsorbs on the fourfold hollow site with the $\mathrm{C}-\mathrm{C}$ axis aligned along the [001] direction, and the other adsorbs at low-symmetry site with slightly lower binding energy. The valence-band spectra indicated that acetylene is rehybridized slightly beyond $s p^{2} .8$

The adsorbed states of acetylene on $\mathrm{Cu}(110)$ are complicated with preferential aggregation and coexistence of more than one species; a local probe method is indispensable for further understandings of this system. In this paper, we investigate adsorption of acetylene on $\mathrm{Cu}(110)$ by directly imaging individual molecules with STM. The adsorbates preferentially aggregate into islands at $40 \mathrm{~K}$ and a variety of

${ }^{a)}$ Electronic mail: hokuyama@kuchem.kyoto-u.ac.jp clusters such as dimers, trimers, and even larger islands were observed. The adsorption site of an isolated species was determined to be fourfold hollow site. Vibrational characterization by inelastic electron tunneling spectroscopy revealed $\sim s p^{3}$ rehybridization of acetylene. An acetylene molecule was induced to migrate along the surface by tunnel electron from STM. The migration is restricted along the trough and proceeds in two steps: the molecule first hops to the adjacent long-bridge site and then to the next fourfold site. The hopping motion was induced by inelastic electron tunneling that causes vibrational excitation of mainly $\mathrm{C}-\mathrm{H}$ stretch.

\section{EXPERIMENTS}

The experiments were carried out in an ultrahighvacuum chamber with a base pressure below $5 \times 10^{-11}$ Torr, equipped with a commercial STM (Unisoku, USM-1200). The STM images were acquired in constant current mode at $5 \mathrm{~K}$. An electrochemically etched tungsten tip was used as a STM probe. A single crystalline $\mathrm{Cu}(110)$ was cleaned by repeated cycles of sputtering with $500 \mathrm{eV} \mathrm{Ar}^{+}$ions for $15 \mathrm{~min}$ and annealing at $\sim 800 \mathrm{~K}$ for $1 \mathrm{~min}$. The surface was exposed to $\mathrm{C}_{2} \mathrm{H}_{2}$ or $\mathrm{C}_{2} \mathrm{D}_{2}$ gases via a tube doser positioned $\sim 1 \mathrm{~cm}$ apart from the sample surface. The sample was kept at $40 \mathrm{~K}$ during the exposure to rule out a weakly bound species. ${ }^{9}$ Inelastic electron tunneling spectroscopy with STM (STM-IETS) was conducted by positioning the tip just above the center of the molecule, followed by the measurements of current-voltage characteristic, and its first and second derivatives by a lock-in amplifier. ${ }^{2} \mathrm{~A}$ rms modulation of $12 \mathrm{mV}$ at $600 \mathrm{~Hz}$ was used. The tip was automatically repositioned by a tracking routine prior to each voltage ramp, and the spectra were recorded repeatedly for accumulation to improve signal-to-noise ratio.

\section{RESULTS}

Figure 1(a) shows a typical STM image of $\mathrm{Cu}(110)$ exposed to $\mathrm{C}_{2} \mathrm{H}_{2}$ gas. The molecules preferentially aggregate 

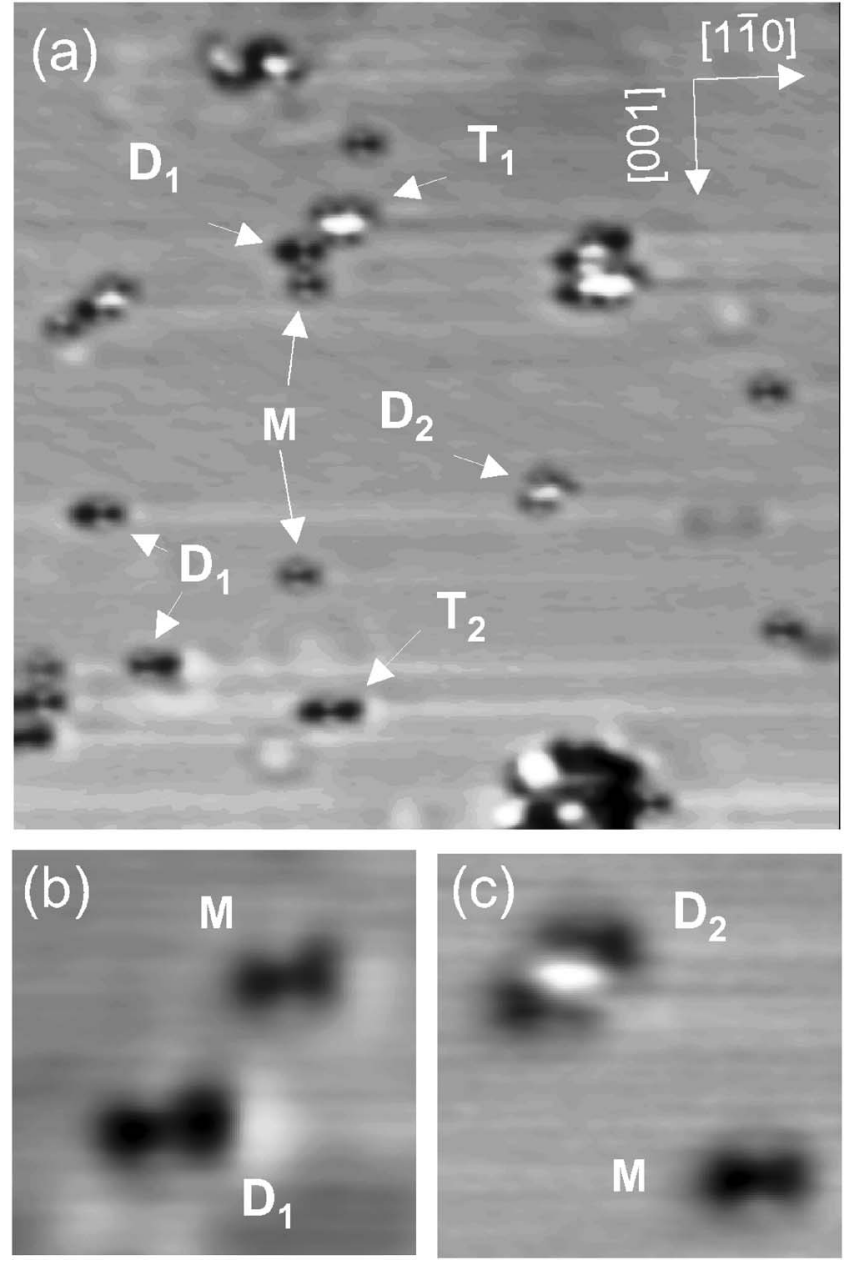

FIG. 1. A typical STM image of $\mathrm{Cu}(110)$ exposed to acetylene $\left(\mathrm{C}_{2} \mathrm{H}_{2}\right)$ at $40 \mathrm{~K}$. (a) A large-scale image $\left(280 \times 280 \AA^{2}\right)$ showing isolated monomer $(M)$, and a variety of clusters such as dimers $\left(D_{1}, D_{2}\right)$, trimers $\left(T_{1}, T_{2}\right)$, and larger islands. $V_{s}=0.1 \mathrm{~V}, I_{t}=0.1 \mathrm{nA}$. Two types of dimer are shown in (b) and (c) in comparison with a monomer $\left(29.5 \times 29.5 \AA^{2}\right)$. (b) The dimer $\left(D_{1}\right)$ appears as an asymmetric dark dumbbell composed of two molecules adjoined along the $[1 \overline{1} 0]$ direction. $V_{s}=-0.1 \mathrm{~V}, I_{t}=0.2 \mathrm{nA}$. (c) The dimer $\left(D_{2}\right)$ consists of two monomers adjoined along the low-symmetry direction with a pronounced protrusion in between. $V_{s}=-0.1 \mathrm{~V}, I_{t}=0.2 \mathrm{nA}$.

into dimers $\left(D_{1}, D_{2}\right)$, trimers $\left(T_{1}, T_{2}\right)$, and even larger islands, which suggests a presence of attractive interaction between the adsorbates. An isolated monomer $(M)$ is observed as a dark dumbbell oriented along the $[1 \overline{1} 0]$ direction, as shown in top right and bottom right of Figs. 1(b) and 1(c), respectively. The appearance is similar to the case of acetylene on $\mathrm{Cu}(001))^{2,3}$ Two types of dimers are shown: one appears as an asymmetric dark dumbbell $\left(D_{1}\right)$ and the other consists of two monomers with a pronounced protrusion in between $\left(D_{2}\right)$. The larger islands consist of depressions and protrusions without any ordered structure.

The appearance depends on the sample bias $\left(V_{s}\right)$ and tunnel current $\left(I_{t}\right)$ as well as the condition of the tip apex. Figure 2 shows typical gap dependence of the STM images for isolated species. The dumbbell-shaped depression is shown in Fig. 2(a). At low gap resistance, an elongated protrusion emerges with a faint dent in the center, as shown in Fig. 2(c), which suggests a presence of a nodal plane in the adsorbate-induced electronic structure involved in the tunnel
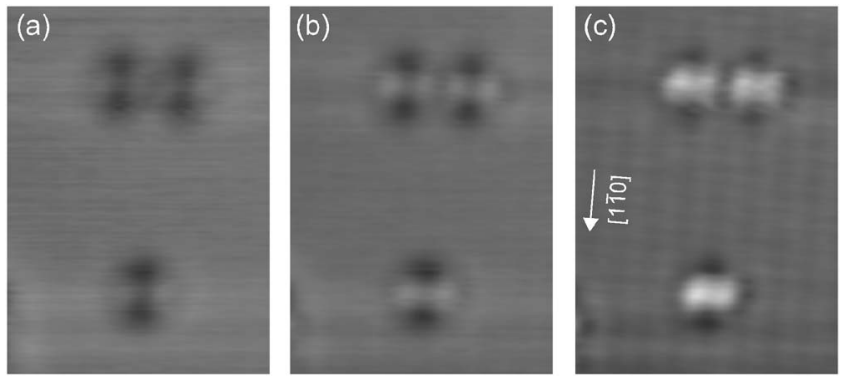

FIG. 2. Typical STM images of three monomers $\left(\mathrm{C}_{2} \mathrm{H}_{2}\right)$ obtained at (a) $V_{s}=-0.25 \mathrm{~V}$, (b) $V_{s}=-0.1 \mathrm{~V}$, and (c) $V_{s}=-7 \mathrm{mV}$ with $I_{t}=0.5 \mathrm{nA}$ (32 $\times 45 \AA^{2}$ ). An elongated protrusion appears in $(\mathrm{C})$ with a faint dent in the center, which reflects the nodal plane of the out-of-plane $\pi^{*}$ orbital of the molecule. The atomic rows are simultaneously observed in (c), indicating that acetylene molecule adsorbs in the trough of $\mathrm{Cu}(110)$.

process. Both the depression and protrusion are comparably observed in Fig. 2(b). We note that the gap dependence as well as the shape of the image is drastically affected by the condition of the tip apex.

The atomic rows are simultaneously observed in Fig. 2(c), and the molecule is determined to be adsorbed in the trough of $\mathrm{Cu}(110)$. Besides, carbon monoxide $(\mathrm{CO})$ was used as a reference molecule to determine the adsorption site. Figure 3(a) shows an image of coadsorbed $\mathrm{C}_{2} \mathrm{H}_{2}$ and $\mathrm{CO}$, which appear as elongated and round protrusions, respectively. In this experiment, the tip apex was probably modified by $\mathrm{CO}$, and thus individual $\mathrm{CO}$ molecules appear as a protrusion, ${ }^{10}$ while it is normally imaged as a round depression with a bare tip. Since CO molecule is established to be adsorbed at the on-top site, ${ }^{9}$ the adsorption site of acetylene can be determined from the relative position. The relation can be represented by integers plus one-half of $a_{0}$ and $b_{0}$ along the [1 $\left.\overline{1} 0\right]$ and [001] directions, respectively. We hereafter represent the atomic distances along [1 $\overline{1} 0]$ (parallel to the row) and [001] (perpendicular to the row) by $a_{0}(2.56 \AA)$ and $b_{0}(3.62 \AA)$, respectively. The adsorption site is thus determined to be fourfold hollow site, as schematically depicted in Fig. 3(b). The orientation of acetylene will be discussed later.

Figure 4(a) shows STM-IETS spectra obtained from an
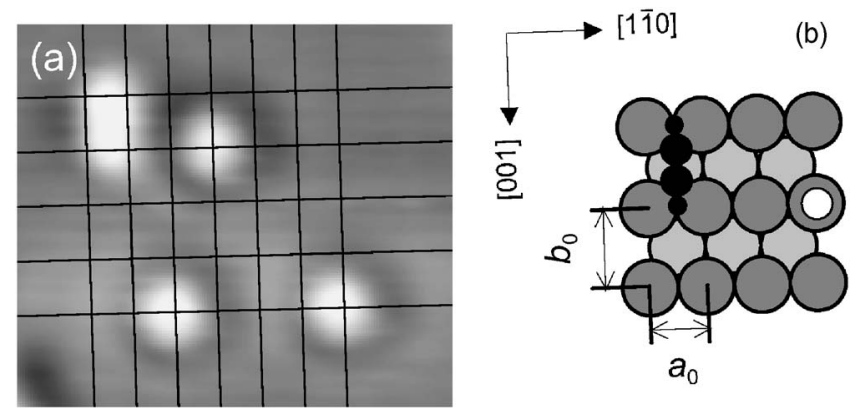

FIG. 3. (a) A STM image of acetylene $\left(\mathrm{C}_{2} \mathrm{H}_{2}\right)$ coadsorbed with carbon monoxide $(\mathrm{CO})$ at $V_{s}=-0.15 \mathrm{~V}$ and $I_{t}=5 \mathrm{nA}\left(23 \times 29 \AA^{2}\right)$. The former appears as an elongated protrusion along the [001] direction, while the latter shows round protrusion. $\mathrm{CO}$ molecules adsorb at the on-top site and the grid lines are depicted through $\mathrm{Cu}$ atoms of the substrate. The adsorption site of acetylene is thus determined to be fourfold hollow site, as schematically shown in (b). The black and white circles indicate acetylene and $\mathrm{CO}$ molecules, respectively. 


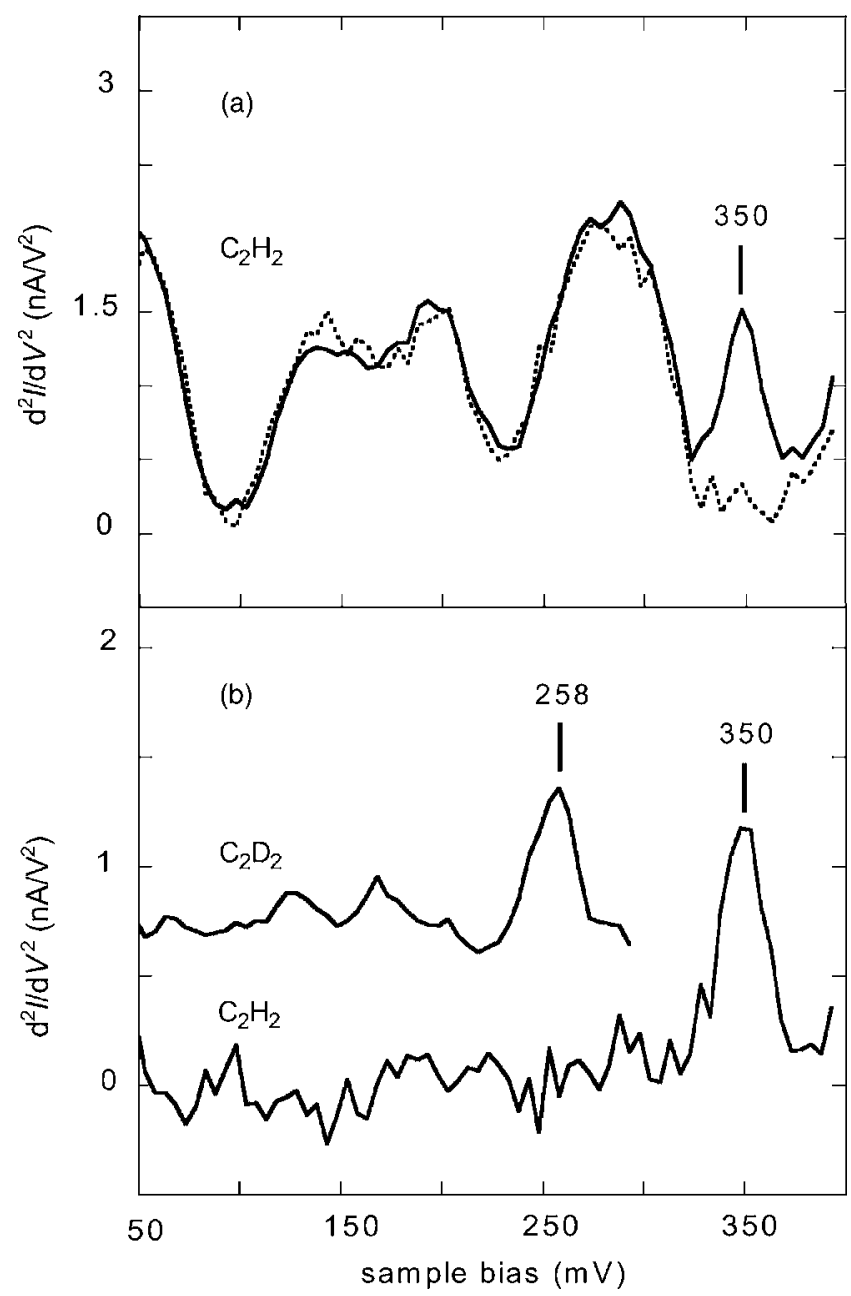

FIG. 4. (a) STM-IETS spectra for an isolated $\mathrm{C}_{2} \mathrm{H}_{2}$ molecule (solid curve) and clean surface (dashed curve). The tip was fixed vertically to give $I_{t}$ $=50 \mathrm{pA}$ at $V_{s}=0.1 \mathrm{~V}$. (b) The differential spectra show peaks at 350 and $258 \mathrm{mV}$ for $\mathrm{C}_{2} \mathrm{H}_{2}$ and $\mathrm{C}_{2} \mathrm{D}_{2}$, respectively, which are assigned to the $\mathrm{C}-\mathrm{H}(\mathrm{D})$ stretch mode of $\sim s p^{3}$ rehybridized acetylene molecules.

isolated $\mathrm{C}_{2} \mathrm{H}_{2}$ molecule (solid curve) and clean surface (dashed curve). The tip was fixed at the center of the molecule and vertically to give $I_{t}=50 \mathrm{pA}$ at $V_{s}=0.1 \mathrm{~V}$. The overall broad features were not dependent on whether a molecule is in the junction or not, but on the condition of the tip apex, and thus it may be ascribed to the electronic state of the tip. The spectrum from a molecule shows a peak at $350 \mathrm{mV}$ which is assigned to the $\mathrm{C}-\mathrm{H}$ stretch mode. The subtracted spectra for $\mathrm{C}_{2} \mathrm{H}_{2}$ and $\mathrm{C}_{2} \mathrm{D}_{2}$ are shown in Fig. 4(b). The isotope shift to $258 \mathrm{mV}$ confirms the assignment of the peaks to the $\mathrm{C}-\mathrm{H}(\mathrm{D})$ stretch vibration. The apparent conductance changes at the $\mathrm{C}-\mathrm{H}$ and $\mathrm{C}-D$ stretch excitations are $3.8 \pm 0.3 \%$ and $3.0 \pm 0.2 \%$, respectively. Since the molecule occasionally hops away from the junction during the repeated voltage ramps as mentioned below, the automatically added spectra give lower estimate of the actual conductance changes. Indeed, we observed a conductance change of $8.0 \pm 0.5 \%$ for a $\mathrm{C}_{2} \mathrm{H}_{2}$ molecule bound in islands (not shown).

It has been reported that STM can induce molecular motions such as rotation ${ }^{3}$ and translation ${ }^{4}$ by injecting energetic tunnel electron to a molecule. In the case of acetylene on
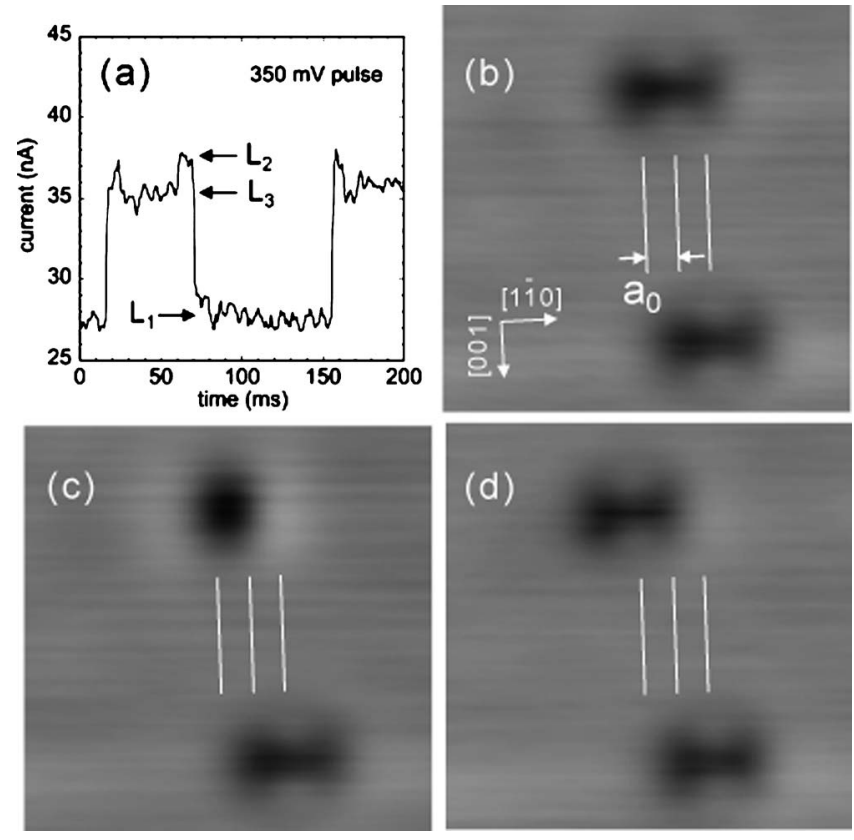

FIG. 5. (a) Tunnel current during a voltage pulse $\left(V_{s}=350 \mathrm{mV}\right)$ over the top molecule $\left(\mathrm{C}_{2} \mathrm{H}_{2}\right)$ in (b). The tip height was fixed to give $I_{t}=8 \mathrm{nA}$ at $V_{s}=$ $-0.1 \mathrm{~V}$ during the measurement. The current jumps at the moment of molecular motion between three discrete levels $\left(L_{1}, L_{2}\right.$, and $\left.L_{3}\right)$, corresponding to the location of the molecule under the tip. The molecule stays at the initial site $\left(L_{1}\right)$ in (b) and hops to the adjacent long-bridge site $\left(L_{2}\right)$ and the next fourfold site $\left(L_{3}\right)$, as shown in (c) and (d), respectively. The lines are depicted with intervals of $a_{0}$ and show the relation between the two molecules along the $[1 \overline{1} 0]$ direction. The images were acquired with $V_{s}=-0.1 \mathrm{~V}$ and $I_{t}=2 \mathrm{nA}$.

$\mathrm{Cu}(110)$, a translational motion is found to be induced with STM. Figure 5(a) shows typical tunnel current during a voltage pulse $\left(V_{s}=350 \mathrm{mV}\right)$ over an isolated $\mathrm{C}_{2} \mathrm{H}_{2}$ molecule. The feedback was turned off during the measurement with the tip fixed vertically to give $I_{t}=8 \mathrm{nA}$ at $V_{s}=-100 \mathrm{mV}$. The current shows jumps between three discrete levels at the moments of molecular motion. At the low level $\left(L_{1}\right)$, the molecule stays just below the tip (the initial state). The pulse was stopped at each current level and STM images were subsequently acquired. As a result, the molecule was found at different sites, as shown in Figs. 5(c) and 5(d), corresponding to the high $\left(L_{2}\right)$ and middle $\left(L_{3}\right)$ levels, respectively. The latter was found off by one atomic distance $\left(a_{0}\right)$ from the original position and thus assigned to an equivalent species at the next fourfold hollow site. In contrast, the former is positioned off one-half atomic distance $\left(0.5 a_{0}\right)$ from the original position, indicating that it is on the long-bridge site in the trough. The appearance is quite different from the original (normal) species with an oval depression accompanied by two protrusions along the trough. This is assigned as an intermediate in the migration process; the low $\left(L_{1}\right)$ and middle $\left(L_{3}\right)$ levels (normal species) are always mediated by the high $\left(L_{2}\right)$ level. We note that the current change between $L_{2}$ and $L_{3}$ is subtle $(\sim 5 \%)$ and it was not discernible in some cases depending on the tip condition. Since the intermediate species was not observed without artificial manipulation (thermodynamically unstable at $40 \mathrm{~K}$ ), it is energetically less favorable (quasistable) than the normal species.

The voltage dependence of the induced motion was in- 


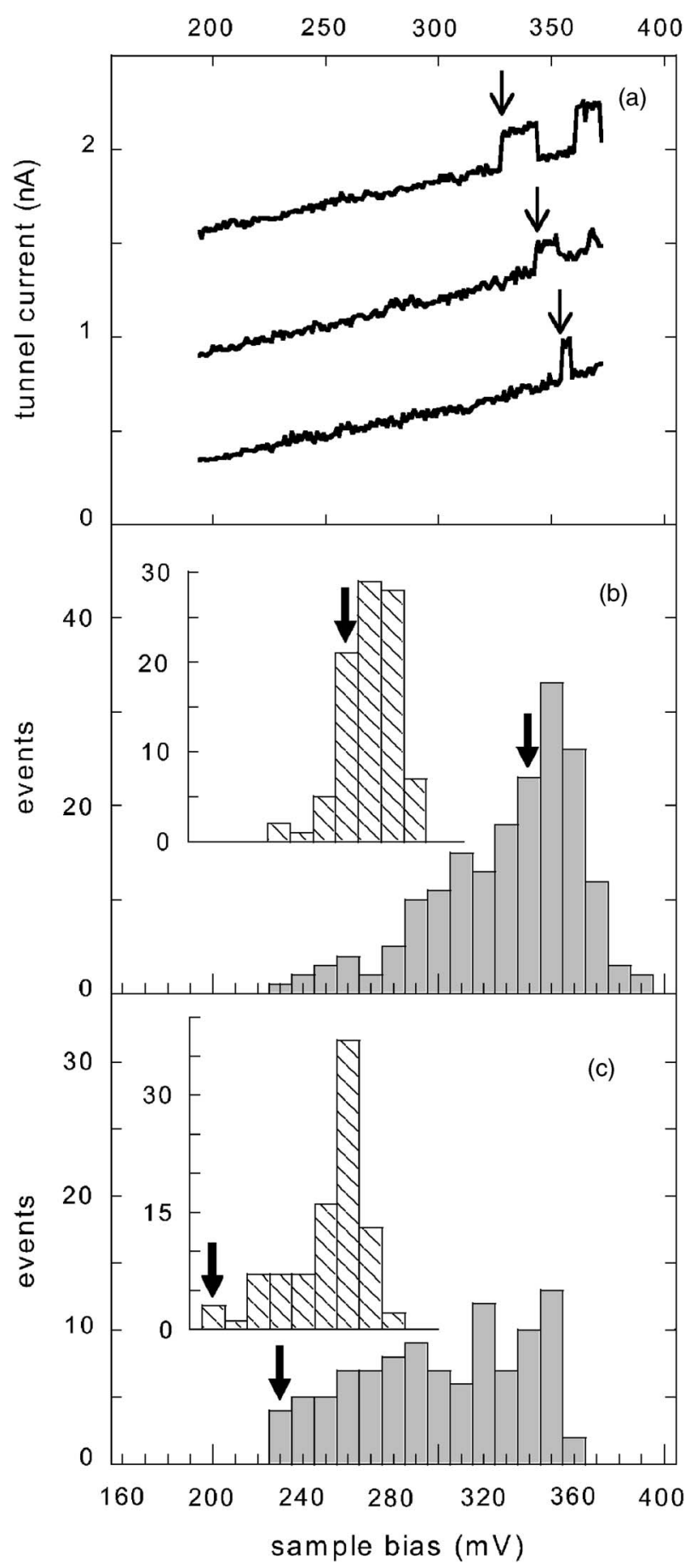

FIG. 6. (a) Typical current-voltage relations during the voltage ramps (20 mV/s) with the tip over a $\mathrm{C}_{2} \mathrm{H}_{2}$ molecule. Tip height was fixed to give $I_{t}=0.3 \mathrm{nA}$ at $V_{s}=-0.1 \mathrm{~V}$. The upper two curves are vertically shifted for clarity. The jumps of the current indicate the moment of the motion. (b) The distribution of the voltages at which the motion was first observed during each voltage ramp for $\mathrm{C}_{2} \mathrm{H}_{2}$ molecules. The inset shows the result for $\mathrm{C}_{2} \mathrm{D}_{2}$ molecules acquired under the same condition. The arrows indicate the approximate thresholds of the $\mathrm{C}-\mathrm{H}(\mathrm{D})$ related process. (c) The distribution similarly obtained at lower gap resistance $\left(I_{t}=3 \mathrm{nA}\right.$ at $\left.V_{s}=-0.1 \mathrm{~V}\right)$. The distributions show mainly two components with threshold voltages around 230 (210) and $340(260) \mathrm{mV}$ for $\mathrm{C}_{2} \mathrm{H}_{2}\left(\mathrm{C}_{2} \mathrm{D}_{2}\right)$. The arrows indicate the thresholds of the lower-energy process.

vestigated in Fig. 6. Figure 6(a) shows typical currentvoltage relation measured over a $\mathrm{C}_{2} \mathrm{H}_{2}$ molecule. During the voltage ramp $(20 \mathrm{mV} / \mathrm{s})$, the feedback was turned off with the tip height fixed to give $I_{t}=0.3 \mathrm{nA}$ at $V_{s}=-0.1 \mathrm{~V}$. A sud-
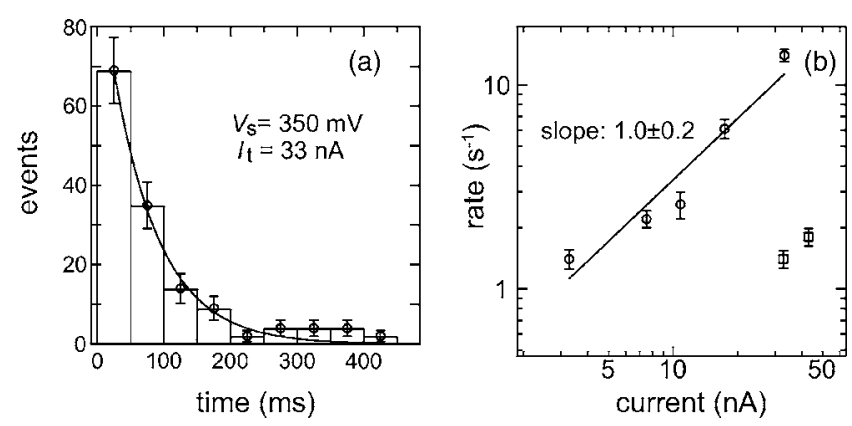

FIG. 7. (a) The distribution of the times the molecule stays just below the tip during the voltage pulse at $350 \mathrm{mV}$. Tunnel current during the pulse was $33 \mathrm{nA}$. The distribution is fitted to an exponential decay from which the hopping rate was deduced to be $(1.4 \pm 0.1) \times 10^{1} \mathrm{~s}^{-1}$. The hopping rates were similarly determined as a function of current (by changing the tip height) and shown in (b) in a logarithmic scale (circles). The slope of $1.0 \pm 0.2$ indicates that the hopping rate is proportional to the current. The result with the voltage pulse at $300 \mathrm{mV}$ is also shown (squares). The hopping rate is $\sim 10$ higher at $350 \mathrm{mV}$ than at $300 \mathrm{mV}$.

den increase of the current indicates the moment of the motion, and the first jump (arrows) occurs above a threshold voltage for the motion (if exists). The high-current state is associated with either adjacent long-bridge or next hollow site, which is not discernible in this experiment. Figure 6(b) shows distributions of the voltage at the first jumps observed for $\mathrm{C}_{2} \mathrm{H}_{2}$ and $\mathrm{C}_{2} \mathrm{D}_{2}$ (inset). The measurement was also conducted at shorter distance $\left(I_{t}=3.0 \mathrm{nA}\right.$ at $\left.V_{s}=-0.1 \mathrm{~V}\right)$, and the distribution is shown in Fig. 6(c). At shorter distance (lower gap resistance), the motion occurs at lower voltage due to higher current. Thus the distribution is dependent on the gap resistance as well as the voltage ramping rate. The histograms suggest two components with threshold voltages around $230(210)$ and $340(260) \mathrm{mV}$ for $\mathrm{C}_{2} \mathrm{H}_{2}\left(\mathrm{C}_{2} \mathrm{D}_{2}\right)$. The latter voltages with clear isotope shift indicate that vibrational excitation of $\mathrm{C}-\mathrm{H}(\mathrm{D})$ stretch is responsible for the induced motion. The former is possibly ascribed to the lower-energy modes such as overtones of $\mathrm{C}-\mathrm{H}(\mathrm{D})$ bending modes.

The current dependence of the hopping motion was investigated in Fig. 7. In a similar way as Fig. 5(a), the current was monitored while the voltage pulse (fixed at $V_{s}$ $=350 \mathrm{mV}$ ) was applied. Figure 7(a) shows the distribution of the times a $\mathrm{C}_{2} \mathrm{H}_{2}$ molecule stays just below the tip at $I_{t}$ $=33 \mathrm{nA}$. It was fitted to an exponential decay, ${ }^{3}$ and the hopping rate was deduced to be $14 \mathrm{~s}^{-1}$. By varying the tip height, the hopping rates were determined as a function of current and shown in Fig. 7(b) in a logarithmic scale (circles). The slope was estimated to be $1.0 \pm 0.2$, indicating that the hopping rate is simply proportional to the current and that the migration is one-electron process. This result supports that inelastic electron tunneling is responsible for the induced motion, rather than electric field under the tip. The hopping rate was also measured at $V_{s}=300 \mathrm{mV}$ in a similar way (squares), which is by a factor of $\sim 10$ lower than that at $V_{s}=350 \mathrm{mV}$.

\section{DISCUSSIONS}

The STM images of an isolated acetylene molecule on $\mathrm{Cu}(110)$ are quite similar to those on $\mathrm{Cu}(001){ }^{2,3}$ The 
dumbbell-shaped depression accompanied by protrusions is commonly observed, and the distance dependence is in qualitative agreement. ${ }^{11}$ The appearance of the image is closely associated with the electronic structure involved in the chemical bonding. Thus the result suggests that the bonding mechanism, e.g., molecular orbital involved, is roughly common between the two cases. According to the calculation for acetylene on $\mathrm{Cu}(001),{ }^{11}$ the $3 b_{2}$ orbital is broadened due to interaction with the substrate and gives contribution to the density of state at the Fermi level $\left(\epsilon_{F}\right)$ and thus to the tunnel current. The notation of the orbital is given under $C_{2 v}$ point group. It is the lowest unoccupied orbital (out-of-plane $\pi^{*}$ ) and is symmetric with respect to the plane including surface normal and the $\mathrm{C}-\mathrm{C}$ axis. Reflecting the spatial distribution of the orbital, the image was predicted to show two protrusions along the $\mathrm{C}-\mathrm{C}$ axis, in agreement with the experiment. ${ }^{2,3}$ Considering the common nature of chemical bonding between $\mathrm{Cu}(001)$ and $\mathrm{Cu}(110)$, we suggest that the protrusion with a dent in Fig. 2(c) reflects the rehybridized $3 b_{2}$ orbital; acetylene molecule adsorbs on $\mathrm{Cu}(110)$ with its axis aligned along the [001] direction. This configuration is consistent with the high-symmetry species proposed by x-ray absorption spectroscopy and density-functional theory. ${ }^{8}$ It was shown that the dumbbell-shaped depression originates from the adsorbate-induced depletion of free-electron-like states of the substrate. ${ }^{11}$

The rehybridization of the adsorbate is studied by vibrational spectroscopy. The IETS spectra show vibrational peaks of $\mathrm{C}-\mathrm{H}(\mathrm{D})$ stretch at 350 (258) $\mathrm{meV}$ for an isolated acetylene molecule, indicating significant rehybridization to $\sim s p^{3}$ on $\mathrm{Cu}(110)$. The electron energy loss spectroscopy (EELS) studies showed $\mathrm{C}-\mathrm{H}$ stretch peaks at $359 \mathrm{meV}$., The discrepancy between the vibrational energies observed by the previous EELS and IETS may result from different surface conditions (temperature and/or coverage). In our preliminary EELS experiment at $110 \mathrm{~K}$, the $\mathrm{C}-\mathrm{H}$ stretch mode was observed at $350 \mathrm{meV}$ without marked dependence on the coverage, in agreement with the IETS. Further systematic study with EELS would settle the discrepancy and determine the symmetry of the IETS active mode.

The histograms in Figs. 6(b) and 6(c) suggest contribution of mainly two processes in the STM induced migration. It is obvious that the $\mathrm{C}-\mathrm{H}$ stretch mode is mainly responsible for the induced motion. The excitation of $\mathrm{C}-\mathrm{H}$ stretch mode was first shown to induce the motion (on-site rotation) of an acetylene molecule on $\mathrm{Cu}(001))^{3}$ The hopping rate is proportional to the current, which supports that the excitation is induced by inelastic electron tunneling. The motions were suggested to result from anharmonic coupling between the high-energy mode (e.g., C-H stretch) and low-energy modes (hindered translation and rotation). ${ }^{3,4}$ We believe this model be applicable to the present case. The lower-energy process can be ascribed to overtone/combination excitations, although its hopping rate is one order of magnitude lower than that for the $\mathrm{C}-\mathrm{H}$ related process. The low rate results in the broad distribution of voltage for the lower-energy process (Fig. 6).

The topographic image of the intermediate species shows oval depression elongated along the [001] direction accompanied by two protrusions at both sides along the trough. It lies at the long-bridge site with the $\mathrm{C}-\mathrm{C}$ axis oriented along either [1 $\overline{1} 0]$ or [001] direction. If we assume that the two protrusions are contributed from the rehybridized $3 b_{2}$ orbital, the $\mathrm{C}-\mathrm{C}$ axis is oriented along the trough, i.e., the hopping motion involves the rotation of the molecule. A theoretical simulation would be required to determine the orientation of the intermediate species.

Based on the absence of a threshold coverage toward trimerization reaction, acetylene was suggested to aggregate into island on $\mathrm{Cu}(110) .{ }^{6,7}$ The STM images directly show preferential aggregation at $40 \mathrm{~K}$. The dimer $\left(D_{2}\right)$ consists of two molecules relatively located $a_{0}$ and $b_{0}$ apart in the [1 $\left.\overline{1} 0\right]$ and [001] directions, respectively. Whenever acetylene molecules adjoin across the atomic row, a pronounced protrusion emerges in between. Similar feature is observed for the trimer in the triangular arrangement $\left(T_{1}\right)$ in Fig. 1(a). This suggests that the density of state near $\epsilon_{F}$ increases at the center of the paired molecules and that the chemical interaction is a candidate for the attractive force. The out-of-plane $\pi^{*}$ orbital of acetylene $\left(3 b_{2}\right)$ is broadened and fractionally occupied by the interaction with the substrate states. ${ }^{11}$ The attractive force originates possibly from the intermolecular coupling of this orbital through the substrate states.

On the other hand, the asymmetric dimer $\left(D_{1}\right)$ consists of two molecules along the trough. The small and large depressions can be attributed to the normal and long-bridge (intermediate) species, respectively, with an interval of $\sim 1.5 a_{0}$. Furthermore, three molecules are arranged along a trough in the bridge-hollow-bridge configuration yielding a trimer $\left(T_{2}\right)$, as shown in Fig. 1(a). The origin of the attractive interaction along the trough may be different from that across the row. We note that the long-bridge species is not present as an isolated species but as a constitute of clusters at $40 \mathrm{~K}$. The larger islands are composed of bright and dark regions without any ordered structure. Although we cannot resolve individual molecules in the island, the preferential aggregation of the normal and long-bridge species along the trough implies that both two species coexist in the island. The coexistence is consistent with the result of $\mathrm{x}$-ray spectroscopic study at $100 \mathrm{~K},{ }^{8}$ apart from the difference in temperature under study. The attractive interaction is closely related to the efficiency in the trimerization reaction on this surface. Thermal evolution of islands would be an essential subject in future study to elucidate the elementary process, such as the presence of an intermediate species. 6.7

\section{CONCLUSIONS}

We have studied adsorption of acetylene on $\mathrm{Cu}(110)$ by imaging individual molecules with STM. A variety of clusters (dimers, trimers, and larger islands) was observed, which is consistent with the previous suggestion. An isolated molecule adsorbs on the fourfold hollow site with the $\mathrm{C}-\mathrm{C}$ axis oriented along the [001] direction. Vibrational characterization by IETS revealed $\sim s p^{3}$ rehybridization of acetylene. An isolated species was induced to migrate along the surface by tunnel electron from STM. The migration is restricted along the trough and proceeds in two steps: the molecule first hops 
to the adjacent long-bridge site and then to the next fourfold hollow site. The voltage and current dependencies of the hopping probability indicate that vibrational excitation (mainly $\mathrm{C}-\mathrm{H}$ stretch) by inelastic electron tunneling is responsible for the induced motion.

\section{ACKNOWLEDGMENT}

This work was supported in part by a Grant-in-Aid from the Ministry of Education, Science, Sports and Culture (Japan).

${ }^{1}$ W. Ho, J. Chem. Phys. 117, 11033 (2002).

${ }^{2}$ B. C. Stipe, M. A. Rezaei, and W. Ho, J. Chem. Phys. 280, 1732 (1998).
${ }^{3}$ B. C. Stipe, M. A. Rezaei, and W. Ho, Phys. Rev. Lett. 81, 1263 (1998).

${ }^{4}$ T. Komeda, Y. Kim, M. Kawai, B. N. J. Persson, and H. Ueba, Science 295, 2055 (2002).

${ }^{5}$ N. R. Avery, J. Am. Chem. Soc. 107, 6711 (1985).

${ }^{6}$ J. R. Lomas, C. J. Baddeley, M. S. Tikhov, and R. M. Lambert, Langmuir 11, 11 (1995)

${ }^{7}$ J. R. Lomas, C. J. Baddeley, M. S. Tikhov, R. M. Lambert, and G. Pacchioni, Langmuir 13, 758 (1997).

${ }^{8}$ H. Ostrom, D. Nordlund, H. Ogasawara, K. Weiss, L. Triguero, L. G. M. Pettersson, and A. Nilsson, Surf. Sci. 565, 206 (2004).

${ }^{9}$ M. Doering, J. Buisset, H.-P. Rust, B. G. Briner, and A. M. Bradshaw, Faraday Discuss. 105, 163 (1996).

${ }^{10}$ L. Bartels, G. Meyer, and K.-H. Rieder, Appl. Phys. Lett. 71, 213 (1997).

${ }^{11}$ F. E. Olsson, M. Persson, N. Lorente, L. J. Lauhon, and W. Ho, J. Phys. Chem. B 106, 8161 (2002). 